\title{
Endovascular management of central venous stenosis after proximal native arteriovenous fistula in hemodialysis patients
}

\author{
Adel H Kamhawy,MD; Mohamed A,MD; El Heneidy,MD; \\ Amr M Abo Rahma,MD
}

Vascular Surgery Unit, Faculty of Medicine, Tanta University, Egypt.

\begin{abstract}
Central venous stenosis (CVS) is a common and significant problem in the long-term management of the hemodialysis access. The aim of this work was to study the efficacy and safety of endovascular management of central venous stenosis in relieving patient's symptoms and maintaining fUnctioning native shunt in end stage renal disease patients on hemodialysis. Balloon angioplasty (PTA) with or without stent were performed for 42 patients who had chronic renal failure and venous hypertension due to CVS. The studied group was 28 males and 14 females, mean age 45 years with 30 brachiocephalic and 12 basilic vein supeificialization. Our patients had 3 SVC, 5 innominate, 34 subclavian vein stenosis. PTA alone was technically successfUl in 27 (64.3\%) of 42 cases. Primary stents were placed in the remaining 15 (35.7\%). All procedures were technically successfUl with no major complications. Primary patency rate were $85 \%, 67 \%$ and $44 \%$ at 3, 6 and 12 months respectively. 28 patients needed reintervention within the 1st year of follow-up. All patients with repeated dilatation had no major complications. In conclusion, Endovascular management of central venous stenosis is an effective and safe alternative to surgery. Follow-up and repeated dilatations are indicated to relieve patient symptoms and extend the life of the proximal native A-V shunt in hemodialysis patients.
\end{abstract}

Introduction:

The growing population of patients with end-stage renal disease (ESRD) and their increased survival has substantially increased the burden associated with dialysis access related complications.! More than $86 \%$ of patients with ESRD are dependent on hemodialysis (HD).2 Management of the complications ofHD access is now integral to vascular practice. 3

Central venous stenosis (CVS) is a common and significant problem in the long-term management of the HD access.4 The placement of catheters in the subclavian veins have led to the development of stenosis in patients up to $40-50 \%, 5,6$ whereas catheterization of the right internal jugular vein is associated with the lowest frequency of CVS. ${ }^{1}$ Most patients who develop a subclavian vein stenosis remain asymptomatic. Only $1 \%$ to $5 \%$ of these will develop clinically significant stenosis, manifested as venous hypertension in the affected extremity.7 CVS can also occur in the venous outflow of an AVF without previous catheterization. 8

The pathophysiology of CVS is not well known but it is thought to be due to thrombus formation from direct intimal trauma at the catheter site, in turn leading to neointimal fibroplasia.9 Formation of subclavian vein thrombosis does not produce a clinically significant syndrome in most patients because of the rapid development of collaterals bypass the occluded venous segment. But when an AVF is created ipsilateral to the stenosis, the increase in blood flow becomes greater than the capacity of the collaterals, and venous hypertension results. 2 Patients with venous hypertension present with pain, massive edema with or without skin breakdown, and disability of the affected extremity. In addition, the increased venous pressure produces inefficient dialysis. This scenario represents challenging problem to the treating physician.2

Historically, the only treatment option available was ligation of the arteriovenous shunt.1O This is a simple surgical solution and resolves the symptoms of venous hypertension. 
But ligation eliminates a precious site of vascular access for life-sustaining dialysis and precludes future access sites from being created in the affected extremity. 2

Nowadays, the optimal therapy for symptomatic central venous obstruction is not clearly defined. Both endovascular and open procedures have been used for the treatment of CVS and occlusions.8 Several years ago, angioplasty was the treatment of choice for CVS. Several investigators, however, have reported reduced long term patency after angioplasty of central lesions; as compared with peripheral venous stenosis.1l-13 Surgical options are limited due to the morbidity associated with the exposure and repair of these deep thoracic veins.14

Despite the adoption of"Dialysis Outcomes Quality Initiative Clinical Guidelines" and early description of percutaneous interventions in hemodialysis fistulas, there is a relative paucity of information on percutaneous management of dysfunctional arteriovenous fistulas and outcomes after percutaneous interventions.1s

The aim of this work was to study the efficacy and safety of percutaneous balloon angioplasty with or without primary stenting of CVS in relieving patient's symptoms and maintaining functioning native shunt in end stage renal disease patients on hemodialysis.

\section{Materials and methods:}

Between 1st January 2007 and 31 December 2010, 42 chronic hemodialysis patients with proximal native AVF with CVS were included in this study. The clinical presentations of our patients were one or more of the following: significant arm edema Figure(1), prominent chest wall venous collaterals, prolonged bleeding after needle removal and inefficient dialysis (increased recirculation and high venous pressure) Figure(2).

For each patient, medical and demographic variables, including smoking, diabetes mellitus, and hypertension and risk factors such as central line catheter placement were recorded.

Exclusion criteriain this study were: patients undergoing secondary stenting for failed or suboptimal angioplasty, patients with complete occlusion with inability to cross the lesion with guidewire, recent thrombosis, allergy from the radiocontrast material, congestive heart failure, uncorrectable coagulopathy, presence of acute infection, synthetic AVF and distal AVF in the forearm.

Diagnosis of CVS was confirmed in all cases by venogram through the AVF that revealed significant stenosis in the central veins, which was defined as $50 \%$ or more narrowing of the vein. Intervention was done in the same setting of diagnostic venography. Ultrasound is also important to exclude acute venous thrombosis.

\section{Technique:}

The procedure was done under local anesthesia. The arterialized vein is cannulated $3 \mathrm{~cm}$ or more distal to the anastomosis and away from the sites of dialysis puncture. A small subcutaneous tunnel is made between the skin and vein entry point to facilitate compression and placement of a suture. Intravenous Heparin (5000 units) was given IV at start of the procedure. Pass the guide wire via needle into vein then advance the sheath.

Venography using nonionic contrast \{Ultravist 370) was done to defme the site, length and degree of the stenosis. Manual compression on the site of anastomosis was done to enhance the contrast in large sized central veins. An angled-tip hydrophilic guide wire 0.035 was used to navigate through the stenosis. After crossing the lesion, dilatation was done. The diameter of the balloon was chosen to allow for 10\%-20\% oversize, which usually resulted in a balloon diameter 1 or 2 $\mathrm{mm}$ larger than the diameter of reference segment. The maximum pressure applied ranged from 18 to $20 \mathrm{~atm}$. Inflation time for 3 minutes was performed. If there was resistant stenosis or elastic recoil repeated inflations could be done till disappearance of the balloon waist. After dilatation a completion venogram was done. If there was 30-500/o residual stenosis redilatation using a 1-mm larger balloon was done. If there is a residual stenosis $>50 \%$ a stent should be considered.

After the procedure is complete, withdrawal of the introducer sheath and purse-string suture was taken around the entry point and manual compression to stop bleeding Figure(3). The suture was removed 24 hours after the 
procedure.16 The used balloon was Ultra-thin TM, Diamond TM, (Boston). The used stent was Wallstent, Venous Endoprosthesis with Unistep' Plus Delivery System (Boston Scientific Scimed Inc.).

In the present study, 42 chronic hemodialysis patients with proximal native AVF with CVS were included. Patients' demographics are shown in Table(1). All of them had a native proximal AVF, $30 \quad(71.4 \%)$ were brachiocephalic while $12(28.6 \%)$ were basilic vein superficialization. There were 27 lesions (64.2\%) in the right side while $15(35.8 \%$ ) in the left side. Our patients had 34 subclavian (81\%), 5 innominate (11.9\%) and 3 SVC (7.1\%) venous stenosis and were subjected to PTA with or without primary stent. All patients had a history of ipsilateral subclavian vein catheter. Most of them (28/34) had a history of repeated or prolonged catheterization of the subclavian vein for hemodialysis access.

All procedures were performed percutaneously through the fistula. Initial percutaneous angioplasty was technically successful in $27(64.3 \%$ ) of 42 cases. Suboptimal results from PTA led to stent placement in the remaining $15(35.7 \%)$. Selfexpanding stents were used to successfully treat these 15 residual lesions. One challenging case of subclavian vein obstruction was successfully recanalised through combined approach by passage of two guide wires, one through the AVF and the other through the femoral vein. By using the stiff end of the back of the guidewire the occlusion was passed and
PTA with primary stent was performed successfully Figures(4-6).

The overall technical success rate of PTA with or without primary stent was $100 \%$. The size of the balloon for fmal dilatation was $10 \mathrm{~mm}$ and 12nun according to adjacent normal venous segment. Balloon length was $4 \mathrm{~cm}$ with maximum inflation pressures of 18 to 20 ATM.

No post-operative complications were encountered except access complications in the form of hematoma in one patient. All patients were discharged the day or the day after the procedure. It is to be mentioned that the patency of the vein in the present study was not assessed by objective methods in all patients. The success of the procedure for providing symptomatic relief is the goal of the procedure and ultimately determines procedural success.

The overall one year patency rate of the 42 patients could be successfully followed in 33 patients, for the remaining 9 patients; one died and 5 patients showed loss of the vascular access for haemodialysis due to thrombosis by which they reached the end point of the study and were excluded from the late follow up and 3 patients were missed in the follow up period The mean of follow-up period was 17.4 months (range, 6-39 months). Primary patency rates were $100 \%, 85 \%, 67 \%$ and $44 \%$ at one, 3,6 and 12 months respectively. 28 patients needed reintervention within the 1st year of followup. All patients with repeated dilatation had no major complications.

Table (1): Patients demographics

\begin{tabular}{|l|c|}
\hline \multicolumn{1}{|c|}{ Studied group } & 42 patients with CVS \\
\hline Male & $28(66.6 \%\}$ \\
\hline Right arm & 27lesions $(64.2 \%\}$ \\
\hline Mean age (Y) & 45 years \\
\hline Diabetes & $11(26.2 \%)$ \\
\hline Hypertension & $26(61.9 \%)$ \\
\hline Smoking & $19(45.2 \%)$ \\
\hline One time ipsilateral catheter & $7(16.7 \%)$ \\
\hline Two times or more ipsilateral catheter & $35(83.3 \%)$ \\
\hline AVF in the same limb & \\
\hline Brachiocephalic & $30(71.4 \%)$ \\
\hline Basilic vein superficialization & $12(28.6 \%)$ \\
\hline
\end{tabular}




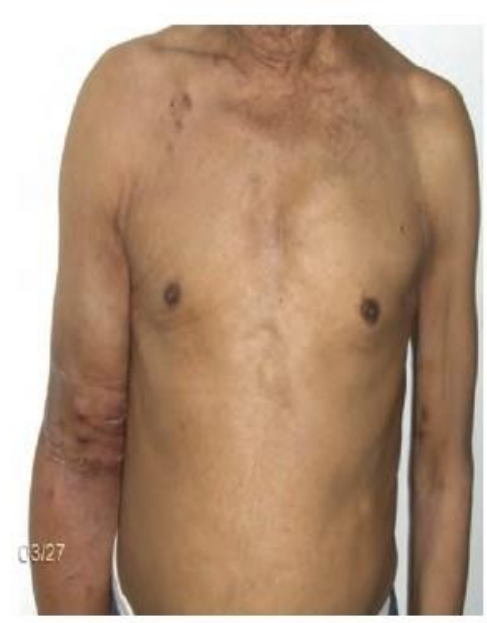

Figure (1): A case of right upper limb edema due to CVS.

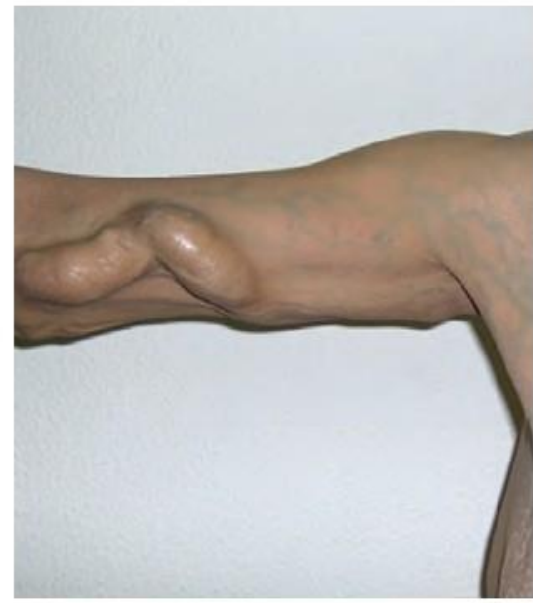

Figure (2): Dilated co/laterals and AVF malfUnction.

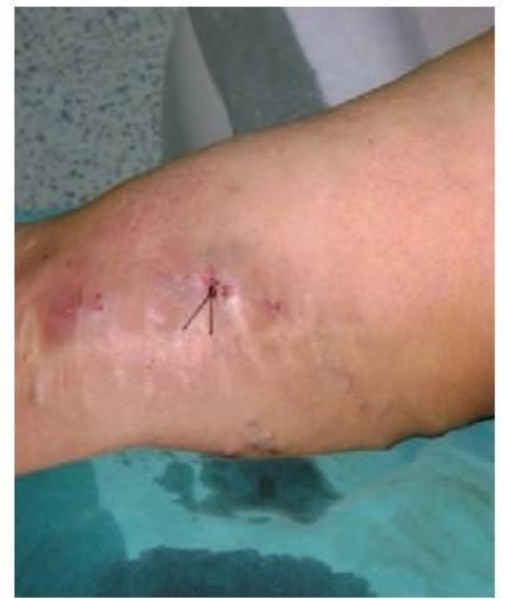

Figure (3): Purse-string suture in the skin tied after extraction of the introducer.

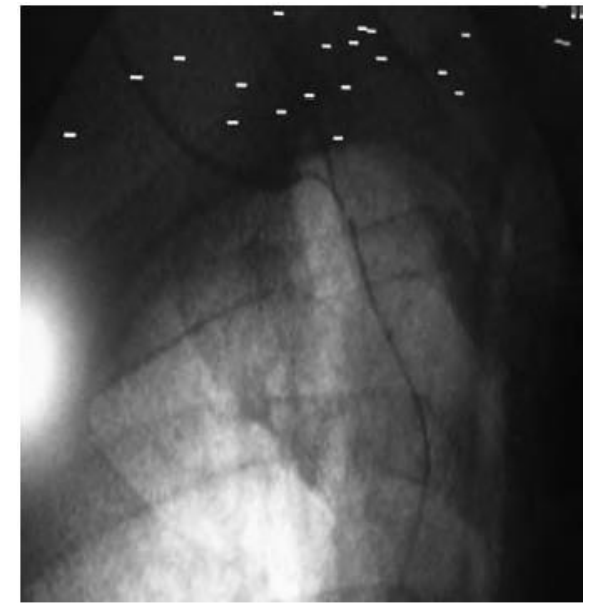

(A)

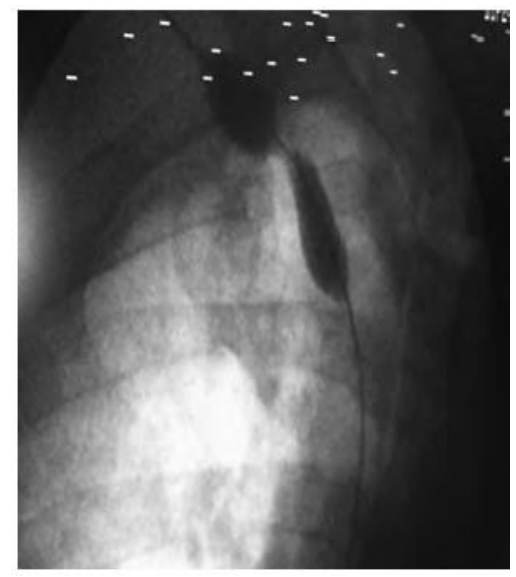

(C)

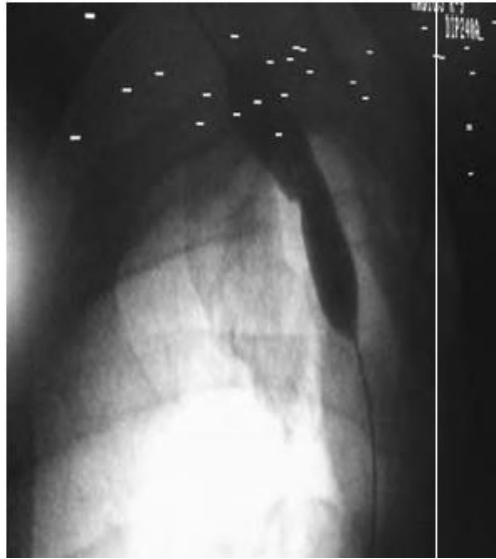

(D)

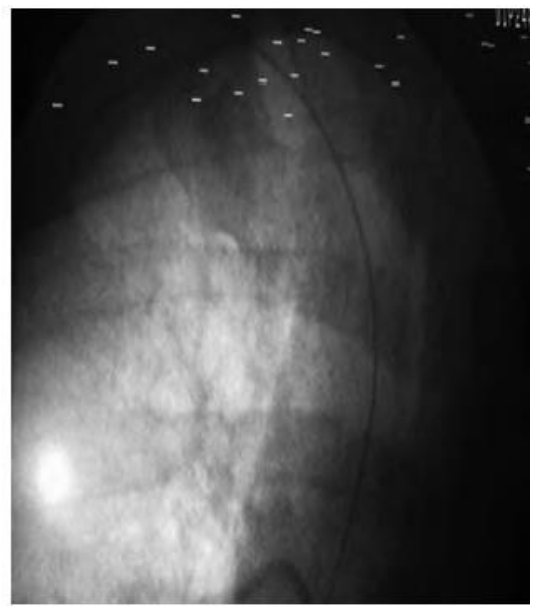

(B)

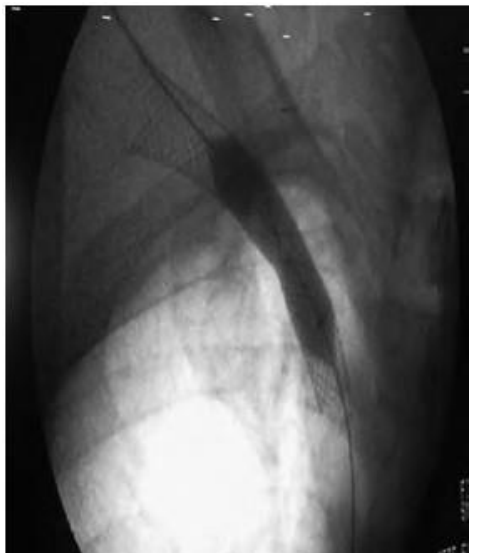

(E)

Figure (4): A case of complete subclavian vein occlusion successfUlly recanalised through combined approach and PTA with primary stent.

(A): Subclavian vein occlusion with two guidewiresfrom above (trans AVF) and below (trans femoral), (B): Successful crossing of the lesion, $(C)$ : Balloon dilatation with waisting, $(D)$ : Complete Balloon dilatation, $(E)$ : Stent placed through the occluded segment. 


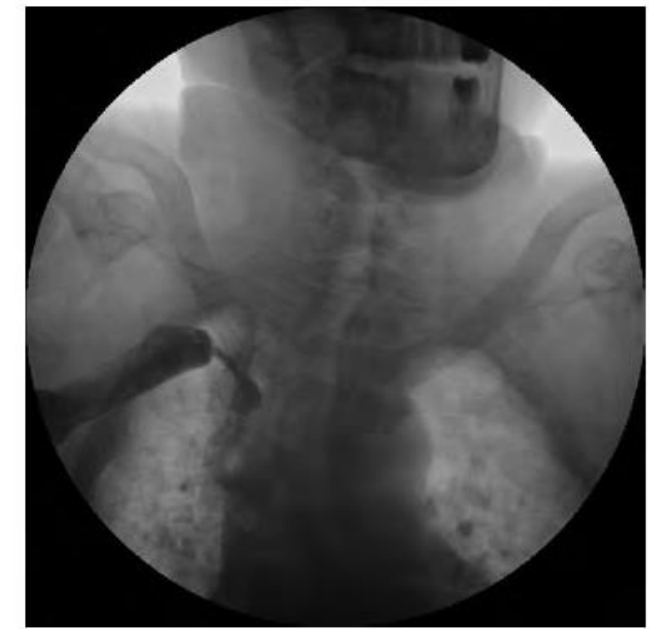

(A)

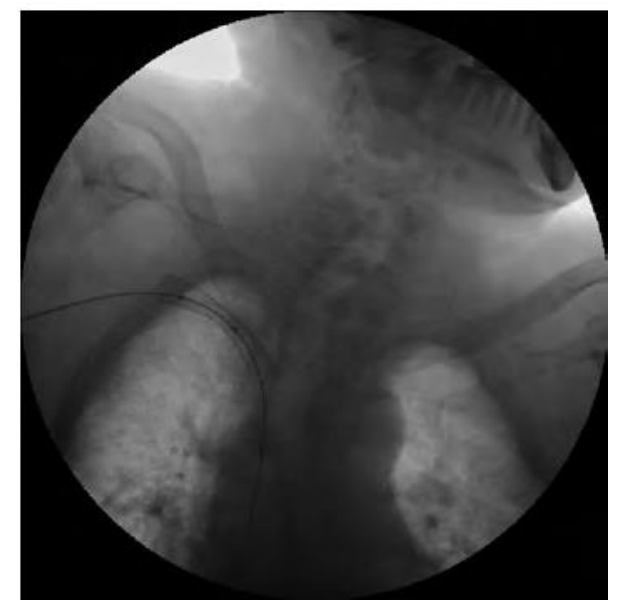

(C)

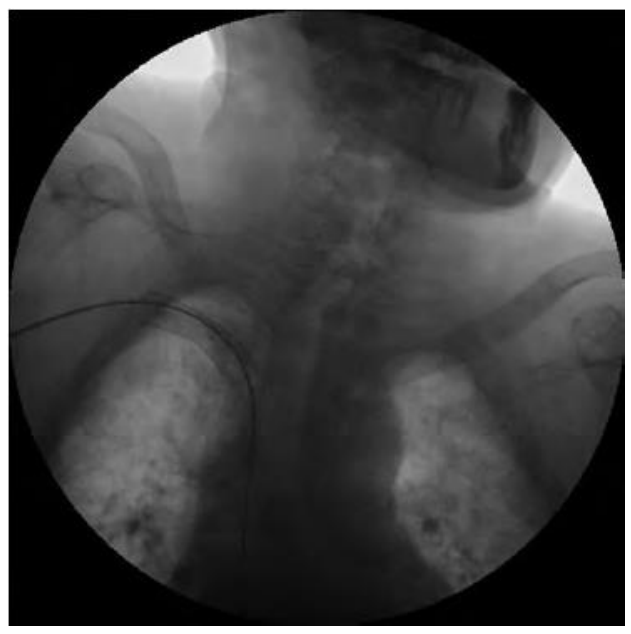

$(E)$

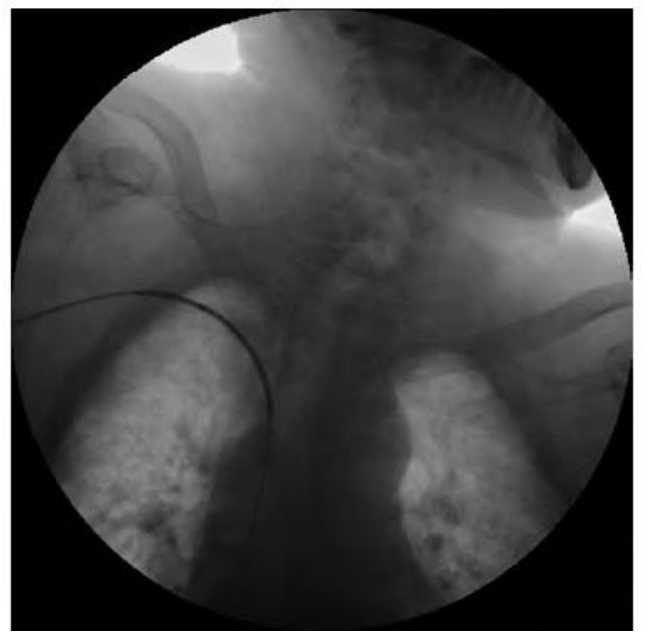

(B)

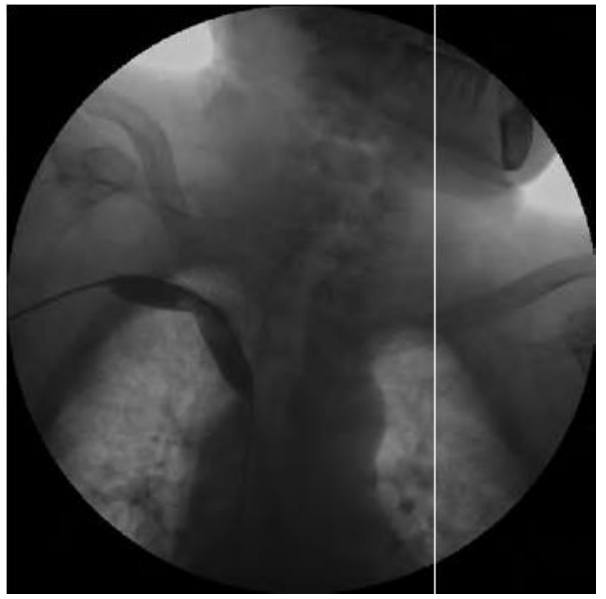

(D)

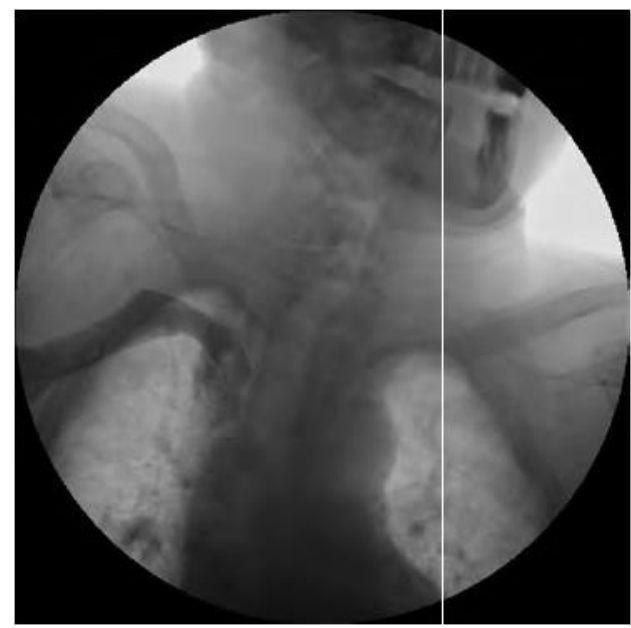

$(F)$

Figure (5): A case of subclavian vein stenosis treated by stent.

(A): Stenosis of Rt subclavian vein, (B): Stent before deployment positioned in place, $(C)$ : Stent after deployment with waisting, $(D)$ : Balloon dilatation inside the stent, $(E)$ : Complete dilatation of the stent, $(F)$ : Complete dilatation without residual stenosis. 


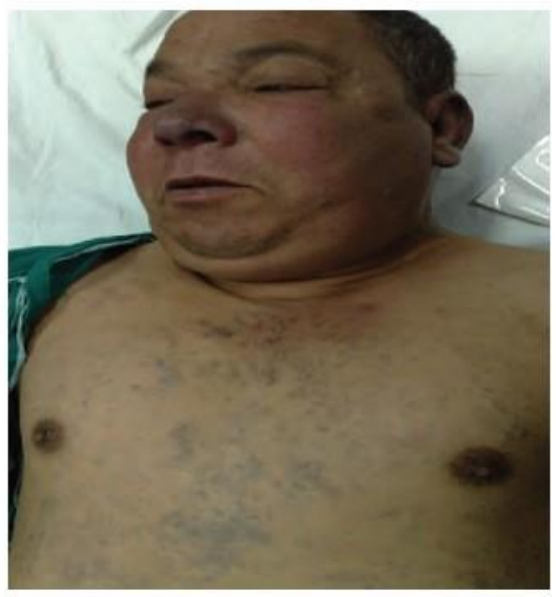

(A)

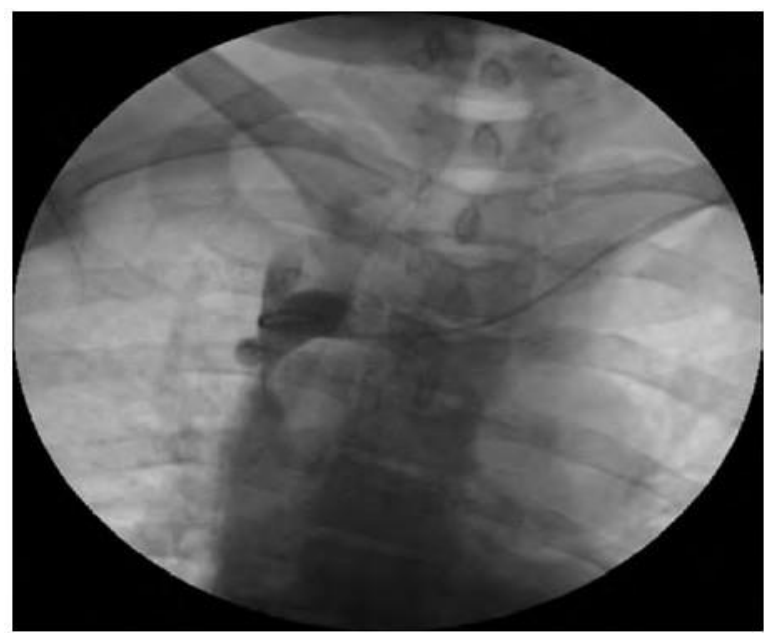

(C)

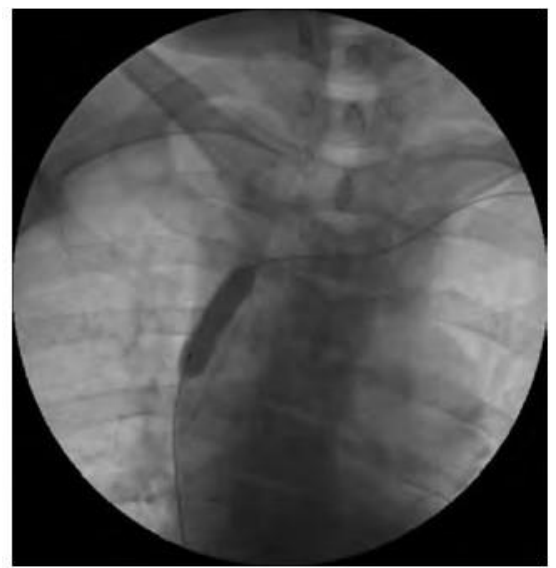

$(E)$

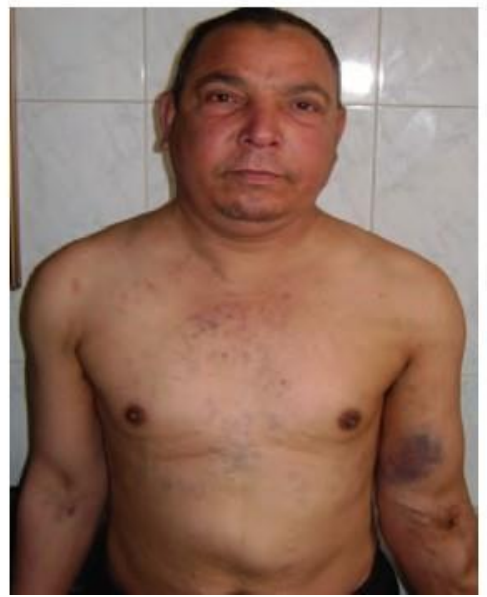

(B)

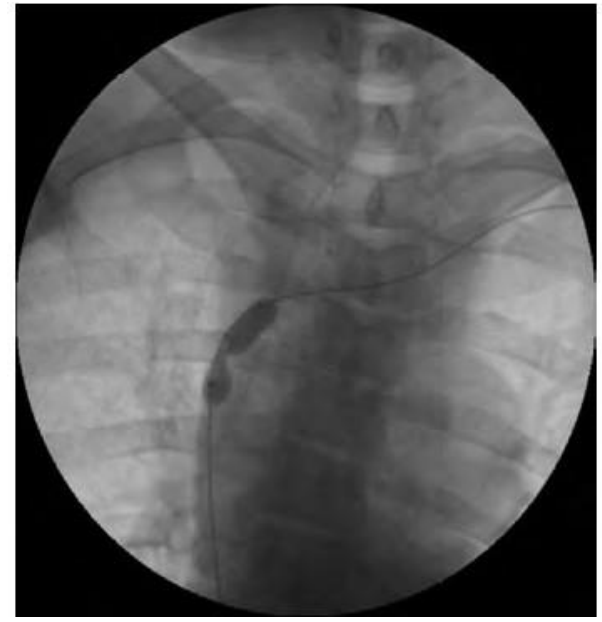

(D)

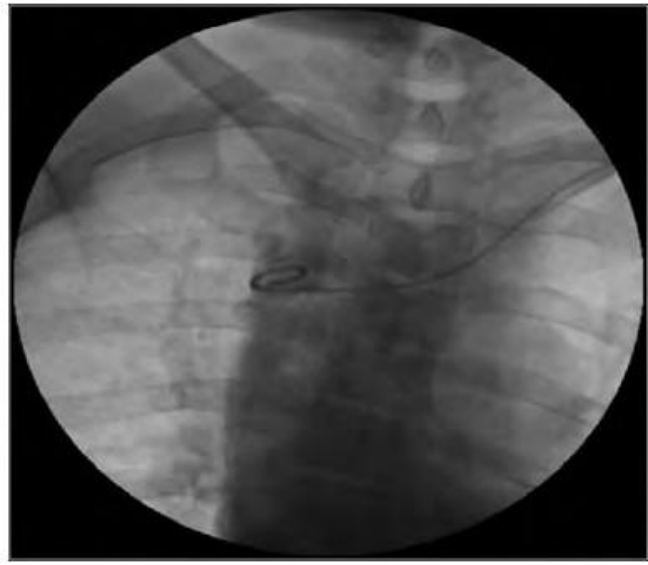

$(F)$

Figure (6): A case of superior vena cava syndrome.

(A): A patient with SVC syndrome with congestion of face and dilated veins over chest wall, (B): Clinical improvement few days after the procedure, $(C)$ : Severe stenosis of $S V C$, $(D)$ : Balloon dilatation with waisting, $(E)$ : Complete dilatation of the balloon, $(F)$ : Venography after complete dilatation with accepted residual stenosis. 


\section{Discussion:}

Percutaneous endovascular methods, including PTA and stent placement, are gaining popularity as therapeutic alternatives to open surgery in treatment of CVS. Endovascular approach can be done under local anesthesia, is well-tolerated by the patient, and is associated with shorter hospitalization time than surgery.14

Because of the steady growth of the HD population and the persistently high percentage of late referrals requiring emergency renal replacement therapy, increasing numbers of HD catheters are being implanted. In Europe between 15\% (Germany) and 50\% (UK) and in the US even $60 \%$ of end-stage renal disease had a history of HD catheters. 17

All patients in this study had a history of prior catheterization which was used in initial dialysis.Inaccordance to other studies, a history of central vein catheter was reported in $40 \%$ \& $90 \%$ in HD patients with CVS_18,19

In our study results analysis clarified that stenosis occurred in areas with no valves like SVC and innominate vein; on contrary to opinion that says stenosis started at the sites of valves by thrombosis then recanalization 20

Concerning the site of entry, in the present study we used catheterization through the fistula. On contrary, Oderich et al.21 preferred catheterization from the femoral venous approach for patients with small-caliber, tortuous, or angulated peripheral upperextremity veins, which could not be accessed through the AVF.

In the current study, the vein most commonly treated was the subclavian vein (34 case- $81 \%$ ), followed by the innominate ( 5 cases-11.9\%) vein and SVC in 3 cases $(7.1 \%)$. The most prevalent lesion type was stenosis rather than occlusion; however most of the stenosis was of the severe type.

In the study of Haage et al.,22 there were an obstruction situated in the subclavian vein in 35 patients out of 50 patients included in his study and in the brachiocephalic vein in 15 patients. Of the 35 patients with obstruction in the subclavian vein, $25(71 \%)$ had a highgrade stricture, and 10 (29\%) had an occlusion. Of the 15 patients with obstruction in the brachiocephalic vein, $13(87 \%)$ had a severe stenosis, and two (13\%) had an occlusion. In another study by Sprouse et al.8 the lesions were most commonly located in the subclavian vein. It was involved in $17(53 \%)$ cases followed by the innominate vein in $11(34 \%)$, axillary vein in $3(9 \%)$, and superior vena cava in $1(3 \%)$.

In the current work, the type of balloon used for all patients were illtra-thin Diamond TM, (Boston Scientific). The maximum pressure applied ranged from 18 to $20 \mathrm{~atm}$. Inflation time was $3 \mathrm{~min}$. Trerotola et al.23 stated that in his long standing practice has been to treat hemodialysis-related venous stenosis in an escalating fashion, beginning with moderately high-pressure angioplasty balloons (Ultra-thin; Boston Scientific, Natick, Mass [rated burst pressure, $12 \mathrm{~atm}$ ], which was succeeded by the Ultrathin SDS; Boston Scientific [rated burst pressure, $12 \mathrm{~atm}$ ]) inflated to atmospheric pressures above the manufacturer recommendation (off-label use, $18 \mathrm{~atm}$ for the Ultra-thin balloon, $21 \mathrm{~atm}$ for the Ultrathin SDS balloon). When a resistant stenosis was encountered at these pressures, they used another high-pressure angioplasty balloon (Blue Max; Boston Scientific [rated burst pressure, $20 \mathrm{~atm}]$ ) inflated to pressures above the manufacturer recommendation (offlabel use, $27 \mathrm{~atm}$ ). Also in the study ofBakken et al.3 the angioplasty balloons used were Bard (Murray Hill, NJ) and Boston Scientific (Natick, Mass), with burst pressures of 8 to 15 atm and operational pressures of 10 to $20 \mathrm{~atm}$.

As regards to proper inflation time, Forauer et al.24 demonstrated that technical success was 4.7 times more likely in the 3 versus the 1 minute inflation group. The 1,3 , and 6 month post intervention patencies were not significantly different between the two groups.

The stents used in our study was Wallstent in all cases. Most studies in the same domain reported the use ofWallstent effectively.3,25 It is preffered because of its flexibility and self-expanding nature. The flared ends promote stability in intravascular placement, preventing not only stent migration but also providing a relatively smooth transition at both ends of the stent conduit. It also can be collapsed for removal if it migrates to the heart or pulmonary bed. 25 
Davidson12 stated that Wallstents are the treatment of choice for PTA failures and early recurrences in central veins. Verstandig et al.26 showed the disadvantages of Wallstent which include foreshortening at the time of placement.

In the present study, the overall technical success rate of PTA with or without primary stent was $100 \%$. PTA was technically successful in $27(64.3 \%)$ of 42 cases. In the remaining 15 lesions (35.7\%) stents were used successfully. Reintervention was needed in 28 patients $(66.6 \%)$ within the 1st year of followup. This was in agreement with the results of other studies. Sprouse et al.8 found that initial PTA was technically successful in $26(81 \%)$ of32 cases. Suboptimal results from PTA led to stent placement in the remaining six (19\%) cases. The overall technical success rate of PTA and PTA combined with stent placement was $100 \%$ and needed reintervention in $63 \%$ of cases. Similarly, Bakken et al.3 reported that the initial treatment of CVS was technically successful in $82 \%$ of the PTA group and $96 \%$ ofthe PTS group. However, residual stenosis was significantly more common with angioplasty (53\%) than with stenting (7\%).

It is to be mentioned that the patency of the vein in the present study was not assessed by objective methods in all patients. Regardless of the patency, however, the success of the procedure for providing symptomatic relief is the goal of the procedure and ultimately determines procedural success. Sprouse et al. 8 rarely perform duplex ultrasound scanning to document patency or occlusion in this setting, because it does not alter the plans for venography to assess for recurrent lesions.

No post-operative complications were encountered in the current study except access complications in the form of hematoma in one patient. All patients were discharged the day or the day after the procedure. Similarly, Sprouse et al.8 reported that patients were typically discharged on the day of the procedure after clinical assessment.

On the basis of the results of the present work, it could be concluded that endovascular management of central venous stenosis is an effective and safe alternative to surgery. Follow-up and repeated dilatations are indicated to relieve patient symptoms and extend the life of the proximal native $\mathrm{A}-\mathrm{V}$ shunt in $\mathrm{HD}$ patients.

\section{References:}

1- Chandler NM, Mistry BM, Garvin PJ: Surgical bypass for subclavian vein occlusion in hemodialysis patients. American College of Surgeons 2002; 194(4): 416-421.

2- Nael K, Kee ST, Solomon H, Katz SG: Endovascular management of central thoracicveno-occlusive diseases in hemodialysis patients: A single institutional experience in 69 consecutive patients. J Vase Interv Radiol 2009; 20: 46-51.

3- Bakken AM, Protack CD, Saad WE, Lee DE, Waldman D L: Long-term outcomes of primary angioplasty and primary stenting of central venous stenosis in hemodialysis patients. J Vase Surg 2007; 45: 776-783.

4- Kundu S: Central venous disease in hemodialysis patients:Prevalence, etiology and treatment. The Journal of Vascular Access 2010; 11: 1-7.

5- Surrt R, Picus D, Hicks M, et al: The importance of preoperative evaluation of the subclavian vein in dialysis access planing. AJR 1991; 156: 623-625.

6- Me Cready RA, Hyde GL, Schartz RW, Mattingly SS: Massive upper extremity edema following vascular access surgery. Ann Vase Surg 1988; 2:75-78.

7- Puskas JD, Gertler JP: Internal jugular to axillary vein bypass for subclavian vein thrombosis in the setting ofbrachial arteriovenous fistula.J Vase Surg 1994; 19:939942.

8- Sprouse LR, Lesar CJ, Meier GH, Parent FN:Percutaneous treatment of symptomatic central venous stenosis angioplasty. $J$ Vase Surg 2004; 39:578-582.

9- Bhatia DS, Money SR, Ochsner JL, et al: Comparison of surgical bypass and percutaneous balloon dilatation with primary stent placement in the treatment of central venous obstruction in the dialysis patient: One-year follow-up. Ann Vase Surg 1996; 10: 452-455.

10-Kahn D, Pontin A R, Jacobson J E, et al: Arteriovenous fistula in the presence of subclavian vein thrombosis: A serious 
complication. Br J Surg 1990; 77: 682.

11-Beathard G: Percutaneous transvenous angioplasty in the treatment of vascular access stenosis. Kidney Int 1992; 42:13901397.

12-Davidson CJ, Newman GE, Sheikh KH, Kisslo K, Stack RS, Schwab SJ: Mechanisms of angioplasty in hemodialysis fistula stenosis evaluated by intravascular ultrasound. Kidney Int 1991; 40: 91-95.

13-Kovalik E, Newman G, Suhocki P, Knelson M, Schwab S: Correction of central venous stenosis: Use of angioplasty and vascular Wallstents. Kidney Int 1994; 45: 1177 1181.

14-Ayala JE, Bellows PH, Ismail N, Cheema ZF, Naoum JJ, et al: Surgical management of hemodialysis related central venous occlusive disease: A treatment algorithm. Ann Vase Surg 2011; 25: 108-119.

15-Eknoyan G, Levin NW, Eschbach JW, Golper TA, Owen WF Jr, Schwab S, Steinberg EP: Continuous quality improvement: DOQI becomes K/DOQI and is updated. National Kidney Foundation's Dialysis Outcomes Quality Initiative. Am JKidney Dis 2001; 37: 179194.

16-Vorwerk D, Konner K, Schurmann K, Gunther RW: A simple trick to facilitate bleeding control after percutaneous hemodialysis fistula and graft interventions. Cardiovasc Intervent radio/1997; 20 (2): 159-160.

17-Pisoni RL, Young EW, Dykstra DM, Greenwood RN, Reeking E, Gillespie B, et al: Vascular access use in Europe and the United States: Results from the DOPPS. Kidney Int 2002; 61: 305-316.

18-Koh KH, Tan C: Central vein stenosis in end stage renal failure patients. $J R$ Coli Physicians Edinb 2005; 35: 116-122. 19-0guzkurt L, Tercan F, Yildirim S, Torun D: Central venous stenosis in hemodialysis patients without a previous history of catheter placement. Eur J Radio/2005; 55: 237-242.

20-Bergan J, Pascarella L: Venous anatomy, physiology, and pathophysiology.In: The Vein Book. Bergan JJ (Editor); San Diego, Calif: Elsevier (Publisher); 1st edn 2007; p. 39-68.

21-Oderich GS, Treiman GS, Schneider P, Bhirangi K: Stent placement for treatment of central and peripheral venous obstruction:A long-term multi-institutional experience. J Vase Surg 2000; 32: 760769.

22-Haage P, Krings T, Schmitz-Rode T: Nontraumatic vascular emergencies: Imaging and intervention in acute venous occlusion.Eur Radio/2002; 12:2627-2643.

23-Trerotola S, Stavropoulos W, ShlanskyGoldberg R, T uite KM, Kobrin S: Hemodialysis-related venous stenosis: Treatment with ultrahigh-pressure angioplasty balloons. Radiology 2004; 231 : 259-262.

24-Forauer AR, Theoharis C: Histologic changes in the human vein wall adjacent to indwelling central venous catheters. JVasc Interv Radio/ 2003; 14: 1163-1168.

25-Perry WA: Image-guided endovascular evaluation and intervention for hemodialysis vascular access. In:Access for Dialysis: Surgical and Radiologic Procedures.Davidson JA (Editor); Landes Bioscience USA (Publisher); 2nd edn. 2002; p. 155-197.

26-Verstandig AG, Bloom AI, Sasson T, Haviv YS, Rubing er D: Shortening and migration of Wallstents after stenting of central venous stenosis in hemodialysis patients. Cardiovasc Intervent Radio/2003; 26: 5864. 